FORMATION Formation emploi

Revue française de sciences sociales

132 | octobre-décembre 2015

Enseignement supérieur et mondes économiques : de nouveaux éclairages

\title{
Les jeunes peu qualifiés : une diversité de positions sur le marché du travail
}

Unskilled young people: a variety of positions on the labor market

Wenig qualifizierte junge Menschen : Vielfalt der Positionen auf dem

Arbeitsmarkt

Los jóvenes poco calificados : una diversidad de posiciones en el mercado de trabajo

Philippe Bregeon

\section{(2) OpenEdition}

Journals

Édition électronique

URL : http://journals.openedition.org/formationemploi/4613

DOI : $10.4000 /$ formationemploi.4613

ISSN : 2107-0946

Éditeur

La Documentation française

Édition imprimée

Date de publication : 30 décembre 2015

Pagination : 139-158

ISSN : 0759-6340

\section{Référence électronique}

Philippe Bregeon, «Les jeunes peu qualifiés : une diversité de positions sur le marché du travail », Formation emploi [En ligne], 132 | octobre-décembre 2015, mis en ligne le 30 décembre 2017, consulté le 30 octobre 2020. URL : http://journals.openedition.org/formationemploi/4613 ; DOI : https:// doi.org/10.4000/formationemploi.4613 


\title{
Les jeunes peu qualifiés : une diversité de positions sur le marché du travail
}

\author{
PhilipPe Bregeon \\ Sociologue, professeur associé à l'université de Poitiers, membre associé du Groupe de \\ Recherches et d'Etudes Sociologiques du Centre Ouest (GRESCO).
}

Résumé

Les jeunes peu qualifiés : une diversité de positions sur le marché du travail

Le parcours de jeunes sortis depuis plusieurs années et sans diplôme du système scolaire fait l'objet d'une enquête qualitative et longitudinale. Prenant appui sur une typologie, cet article met en exergue la diversité des positions des jeunes par rapport au marché du travail secondaire. Il s'agit de mieux comprendre comment et pourquoi ces positions sont plus ou moins contraignantes et intégratives pour ces jeunes.

Mots clés: jeune, BNQ-bas niveau de qualification, non diplômé, insertion professionnelle, trajectoire d'insertion, enquête d'insertion, recherche d'emploi, étude de cas

Abstract

Unskilled young people: a variety of positions on the labor market

The results of this qualitative and longitudinal research that we have to present in this paper are about the process of young people, without diploma, who has left scholar system few years ago. Bulding on a typology, this article compare their positions to understand how and why these same positions seem to be more or less expensive and integrative fort those young.

Keywords: young person, low level qualifications, non graduate, transition from school to work, pathway to integration, school-to-work transition survey, job search, case study

Journal of Economic Literature: J 24, I 32

Traduction : Auteur 
Cet article entend analyser la diversité des rapports au marché de l'emploi des jeunes adultes à bas niveau de qualification entre retrait, hyper volontarisme et recherche d'un équilibre pour amortir la confrontation avec un marché du travail qui leur est hostilee.

Loin de l'image stéréotypée d'individus se complaisant dans l'assistanat, cette contribution montre que l'emploi demeure un puissant organisateur social pour les jeunes peu diplômés.

Malgré tout, comme nous le verrons, la plupart de ces jeunes témoignent de leur adhésion à la norme que représente encore et toujours l'expérience au travail. Rappelant les migrants, en France, qui doivent présenter leur titre de séjour pour attester d'une légitimité à être sur le territoire, ils exhibent volontiers leur CV aux différentes institutions dédiées aux dispositifs d'insertion pour attester de leurs expériences de travail.

Au-delà de leur permettre de gagner un peu d'argent pour subvenir à leurs besoins, l'emploi symbolise souvent, pour eux, l'expérience d'une dynamique, une certaine maîtrise de soi, une utilité sociale, le moyen d'affirmer son existence. A contrario, le chômage renvoie plutôt à une certaine forme d'immobilisme et d'inutilité.

Pour autant, l'observation des modes de vie de ces jeunes révèle une certaine diversité des positions dans le rapport au marché de l'emploi, diversité que cet article ambitionne d'instruire : Comment comprendre la position de repli ou d'hyper engagement chez des jeunes avec des parcours scolaires apparemment assez proches? Comment ces jeunes intériorisent, plus ou moins, leurs désavantages par rapport au marché du travail. En quoi le maintien d'une certaine dynamique relationnelle dans l'expérience du chômage peut constituer une ressource dans la recherche d'emploi ? Enfin, le chômage et la précarité conduisent-ils mécaniquement à l'affaiblissement des liens sociaux et à la perte du sens de l'existence?

Ainsi, du printemps 2008 au printemps 2011, pendant environ trois années, nous avons rencontré, au minimum une fois par an, 23 jeunes tous sortis sans diplôme du système scolaire. Ce groupe comprend douze jeunes femmes et onze jeunes hommes de nationalité française, nés entre 1978 et 1989 ( $c f$. encadré $\mathbf{1}$ ).

\footnotetext{
1. Cet article rend compte d'une partie des résultats d'une enquête qualitative et longitudinale, en sociologie, au sein d'une équipe de recherches du GRESCO (Groupe de Recherches et d'Etudes Sociologiques du Centre Ouest) de l'université de Poitiers, dans le cadre d'un programme intitulé « Parcours » et financé par le contrat de projet État-Région 2007/2013.
} 


\section{Encadré 1 : Méthode de l'enquête, contexte et population enquêtée}

Pour trouver un échantillon de jeunes, nous avons d'abord sollicité deux organismes de formation qui avaient mis en œuvre des stages d'insertion ciblant les non-diplômés, ainsi qu'une Mission Locale.

Vers la fin de l'enquête de terrain, nous avons rencontré à nouveau ces interlocuteurs institutionnels pour croiser et compléter les informations que nous avions recueillies auprès des jeunes. II s'agissait essentiellement de compléter les curriculums et de lever des incertitudes concernant les formations suivies et les emplois occupés depuis leur sortie du système scolaire.

Dans une démarche compréhensive en direction de ces populations, la proximité avec les institutions, durant l'enquête de terrain, accroît les risques de biais compte tenu, en particulier, des effets d'affiliation dans des dispositifs ou de refus de l'affiliation. C'est pourquoi, dès le début de notre enquête, nous avons privilégié le domicile des enquêtés eux-mêmes comme espace de rencontre.

Lors des entretiens enregistrés, il s'agissait de reconstruire avec eux leurs parcours depuis leur naissance, sous différents aspects : familial, résidentiel, scolaire, matrimonial, institutionnel, professionnel, rapport au marché de l'emploi, etc.

Pour analyser les trajectoires professionnelles, nous avons effectué un repérage des statuts occupés, mois par mois, depuis leur sortie du système scolaire, au regard du travail et de l'emploi.

Nous avons alors identifié treize statuts : travail non déclaré, décrochage par rapport à l'emploi et/ou au travail, demandeur d'emploi, stage d'insertion, CDD (contrat à durée déterminée) aidé, hors Structure d'Insertion par l'Activité Economique, CDD en Structure d'Insertion par l'Activité Economique, Formation qualifiante, Contrat d'apprentissage, Intérim, CDD, CDI (contrat à durée indéterminée) à temps partiel, CDI à temps complet, Auto entrepreneur.

D'un rendez-vous à un autre, il convenait ensuite d'enregistrer au plus près, et pour chacun, les événements les concernant : la situation économique, le mode de vie, les relations avec les institutions ou avec le marché de l'emploi ont-ils connu des évolutions ? II s'agissait d'observer leurs espaces et modes de vie.

Ne prétendant pas à des résultats généralisables, la méthode qualitative et longitudinale doit mettre au jour, de manière fine, les processus dans les différentes carrières. Elle doit permettre aussi de mieux comprendre comment le sens conféré par des personnes à leurs parcours et à leurs pratiques contribue à orienter leurs trajectoires.

Cette enquête a été réalisée en France, sur l'agglomération d'une ville moyenne d'environ 100000 habitants, à distance des grandes métropoles. Le taux de chômage est légèrement inférieur à la moyenne nationale, l'activité est principalement orientée vers les services et le tertiaire. L'espace urbain d'une vie provinciale relativement calme, avec des institutions qui résistent ${ }^{*}$ ), rompt avec la configuration d'environnements stigmatisés, cibles de bon nombre d'études sur les populations en bas de l'échelle sociale.

$\left(^{*}\right)$ : Cette résistance reste relativement vivace avec des associations comme, par exemple : un réseau d'entreprises d'insertion, des équipes de prévention, des maisons de quartier qui réussissent encore aujourd'hui à maintenir du lien social auprès de ces jeunes à bas niveau de qualification. Par maintien du lien social, nous entendons la capacité de ces associations à apporter un minimum de réponses à l'absence d'emploi durable via des emplois aidés, des activités précaires, des stages, des formations. Par ailleurs, la plupart de ces jeunes demeure en relation avec des intervenants sociaux. 
Les parcours scolaires de ces jeunes composent une mosaïque : trois ont terminé leur parcours scolaire dans des établissements spécialisés dans le champ du handicap (IME - Institut médico-éducatif ou ITEP - Institut thérapeutique éducatif et pédagogique), huit en lycée professionnel, deux en Maison familiale rurale, sept en SEGPA - Sections d'enseignement général et professionnel adapté -, trois au collège, hors SEGPA.

Au début de l'enquête, celui dont l'entrée sur le marché du travail est la plus récente a quitté le système scolaire depuis 36 mois, le plus ancien depuis 154 mois, avec une durée moyenne de 75 mois. Ainsi, il s'agit de cheminements longs mais sans forcément d'horizon temporel commun.

Les propos qui suivent visent à caractériser et à comprendre leurs positions vis-à-vis du marché de l'emploi et de la recherche de travail, grâce à la construction d'une typologie $(C f$. tableau sur la caractérisation des parcours des jeunes, en annexe de la version électronique de l'article) :

- "Les intégrés » ont trouvé une relative stabilité socio-économique et une intégration professionnelle ; ils sont trois.

- "Les déconnectés" sont depuis un certain temps hors du marché du travail ; ils sont trois.

- "Les attentistes » adoptent des stratégies d'évitement, sans être formellement déconnectés du marché du travail ; ils sont cinq.

- «Les hyper volontaristes " sont suspendus de manière ostentatoire à un certain idéal de l'engagement dans la recherche d'emploi ; ils sont quatre.

- "Les empêchés " ont intériorisé leur handicap² à cause de nombreux désavantages ; ils sont trois.

- «Les autonomes ", dont la vie sociale est moins altérée que chez les autres par les enjeux de l'accès à l'emploi et la précarité ; ils sont cinq.

Notre démarche de construction d'une typologie a été inspirée, en particulier, par l'ouvrage de Michel Messu (1991), Les assistés sociaux. Analyse identitaire d'un groupe social. ${ }^{3}$

Comme l'indique Messu en référence à Weber, construire une typologie ne signifie pas exposer le réel. C'est faire émerger les discriminants d'un type à un autre pour établir un tableau qui exprime le réel de manière univoque et homogène (Goffman, 1973).

2. Le vocable handicap correspond, dans cet article, à une limitation d'activité et de participation à la vie en société, au sens de l'article 2 de la loi du 11 février 2005 pour l'égalité des droits et des chances, la participation et la citoyenneté des personnes handicapées.

3. Pour autant, notre analyse ne mobilise pas de manière centrale le concept d'assistance qui parait particulièrement réducteur au regard des capacités d'autonomie des jeunes ciblés et occulte la complexité de leurs modes de vie. 
La présentation d'une typologie a sans doute comme inconvénient de suggérer quasiment des positions quelque peu figées, alors qu'il s'agit le plus souvent de phases au fil des « carrières " de demandeur d'emploi.

L'enquête longitudinale nous a souvent permis d'enregistrer des changements de position chez un même individu, d'un rendez-vous à un autre. Tel jeune avait précédemment délivré des propos volontaristes sur l'importance de l'engagement dans la recherche d'emploi. Un an plus tard, il semble beaucoup plus circonspect.

Pour autant, si les déplacements d'une position à une autre peuvent trouver un début d'analyse pour les hyper volontaristes, nous manquons d'indices pour apporter un éclairage sur les autres scenarios, et c'est sans doute une des limites de cet article.

Cette typologie (avec les vocables "intégrés ", " déconnectés ", etc.) ne doit pas être interprétée dans le sens d'un ordre moral, d'une éthique du bon demandeur d'emploi. Pris dans l'expérience récurrente de la précarité, la plupart de ces jeunes recherche légitimement la position la moins contraignante du point de vue de leur équilibre personnel à chaque moment de leur trajectoire. Cette enquête révèle d'ailleurs les limites de la position "d'hyper volontariste".

\section{1 «Les intégrés » et « les déconnectés » : des positions aux extrêmes}

Certains de ces jeunes sans diplôme ont accédé, depuis quelques années, à des situations professionnelles relativement pérennes, le plus souvent parce qu'ils ont pu, à un moment donné, se qualifier. Ce sont " les intégrés ", et ils correspondent à la petite minorité de notre population.

Il s'agit par exemple de Charlène, à présent titulaire à la poste, depuis la fin de son apprentissage de factrice et l'obtention de son CAP (certificat d'aptitude professionnelle) à un peu plus de 21 ans.

Depuis sa titularisation, elle a quitté l'appartement de sa grand-mère en centre-ville, avec qui elle vivait depuis l'enfance, pour prendre un logement avec son compagnon qui travaille dans une enseigne de distribution de produits culturels. Bien qu'assez modeste, leur situation économique ne semble pas représenter un problème pour eux. Charlène a développé son réseau relationnel, en particulier avec certains collègues de La Poste et des proches de son compagnon. Ils envisagent d'attendre quelques années avant d'avoir un enfant.

Son discours évoque les conditions de travail. Il est question des contraintes et des avantages de son emploi. L'appartenance au monde du travail s'élabore en particulier avec la 
conscience d'une carrière à construire. Ainsi se dessine une intégration apparemment assez traditionnelle au sein d'un salariat d'employés.

Au sortir du système scolaire, la phase de recours aux dispositifs d'insertion a duré environ deux années. Aujourd'hui, elle en parle avec un certain détachement : "Pour moi, c'est une période loin derrière moi, que j’ai déjà un peu oubliée. Sans doute, ça m’a un peu aidée à une période où je ne savais pas trop quoi faire. Pour autant, il ne faut pas trop s'installer dans ce genre de système, il faut surtout s'appuyer sur soi-même et sur les amis. Moi, c'est par les amis que j'ai trouvé à la Poste."

On retrouve en général une condamnation tacite d'un usage prolongé des dispositifs d'insertion.

Ainsi, le point de vue des « intégrés » met en avant la primauté de la sphère privée comme clé de l'intégration socioprofessionnelle : si les dispositifs ont pu avoir quelques effets positifs sur leur parcours, c'est parce qu'il y avait juste un petit coup de pouce à donner et que la collaboration a été assez brève.

A contrario, une faible minorité des enquêtés se maintient durablement hors du marché du travail. On peut les dénommer "les déconnectés ".

Il s'agit, en particulier, de jeunes femmes qui reproduisent, à leur insu, le modèle traditionnel de la femme au foyer, pour élever les enfants en bas âge. Il ne s'agit pas réellement d'un choix, mais plutôt d'un repli.

Ainsi, à 22 ans, et après une scolarité en IME, Amélie est mariée depuis peu avec Mokhafi, d'origine maghrébine, "en attente de régularisation ». Elle reste pour l'instant à la maison pour s'occuper de son bébé, mais espère pouvoir travailler dans quelques années. Elle hésite sur le domaine d'activité :

"Je ne sais pas encore ce que je vais faire plus tard... Peut-être le travail en maison de retraite ou alors coiffeuse ou dans la vente de prêt-à porter..."

L'insertion dans le marché du travail est renvoyée à plus tard, dans une forme souvent assez idéalisée, avec l'espoir de pouvoir affirmer son identité par le travail.

Née en France de parents français, elle est dans l'immédiat mobilisée par sa conversion récente à la religion musulmane et sa décision de porter le voile, qu'elle exhibe un peu comme un étendard.

Juliana, mariée, reste elle aussi à la maison. Depuis la naissance de son premier enfant, il y a trois ans, elle est assistante maternelle et garde un enfant en plus de son bébé :

"Je passe la journée avec les enfants, je joue, je sors avec eux parfois quand il fait beau, c'est très bien comme ça..."

A l'opposé d'une majorité qui mène une recherche active, ces positions représentent les jeunes les plus éloignés de l'emploi salarié. 


\section{La position des « attentistes »}

Entre les deux positions précédentes, on trouve, entre autres, les attentistes : ceux qui ont appris à mettre à distance la question de l'emploi tout en restant connectés, à leur manière, au marché du travail. Les premières expériences de socialisation professionnelle semblent avoir marqué durablement le rapport à l'emploi.

Nestor a commencé son parcours professionnel, il y a environ six ans, par un apprentissage en peinture, interrompu au bout de quelques semaines : "La première semaine chez ce patron, j'étais avec un employé sur le chantier, puis après, ils me laissaient tout seul... Je faisais de la pose de bandes de Placoplatre sur des maisons toutes neuves. Au bout de quelques semaines, j'en avais marre d'être seul et le patron a dit que je n'allais pas assez vite, il fallait avoir un rendement. Mais il n'était pas très honnête, parce qu'il n'a jamais fait les papiers pour me déclarer. Dans ces conditions, c'était mieux d'arrêter..."

Entre les périodes de recherche d'emploi sans grande conviction, Nestor et Roger s'octroient « des respirations » prolongées.

Ils ont tous les deux quitté le système scolaire après une année en classe « de préapprentissage ", sans avoir trouvé d'entreprise. Au cours des années suivantes, ils ont alterné l'un et l'autre de longues périodes de chômage avec quelques stages d'insertion.

Ils font un peu de trafic ${ }^{4}$ de cannabis pour assurer leur propre consommation et se faire un peu d'argent.

A la différence de Nestor, considéré par la Mission Locale et l'équipe de prévention comme proche de l'illettrisme, Roger a pu suivre, ces dernières années, deux formations qualifiantes avec l'AFPA (Association nationale pour la formation professionnelle des adultes) : une formation de façadier d'abord, puis de coffreur-bancheur, pour laquelle il a obtenu la qualification, il y a environ un an.

Depuis, il n'a toujours pas trouvé de travail et, dans la mesure où il est à présent doté d'une qualification, cette situation génère des tensions avec son conseiller de la Mission Locale qui met en cause sa motivation par rapport au marché de l'emploi.

Roger entend lui montrer qu'il a effectué quand même des démarches ces derniers mois pour trouver un emploi : "J'ai envoyé un $C V$ et une lettre de motivation à l'entreprise $B$ dans le bâtiment qui m'a répondu qu'il n'y a rien pour l'instant, et je me suis inscrit en intérim, donc, jattends..."

4. Selon plusieurs institutions en lien avec eux. 
Cependant, il justifie son chômage par les difficultés du marché du travail : «En intérim, c'est toujours le même truc quill racontent : ces temps-ci, c'est la crise!"... "Dans cette ville, quoi que l'on fasse pour trouver un boulot, j'ai l'impression quil n'y a rien..."

Il justifie à sa manière son peu de motivation par rapport aux métiers du bâtiment, pour lesquels il est maintenant formé: "Dans le bâtiment, il faut se défoncer au travail pour suivre les autres... On rentre le soir et souvent, on est complètement cassé. C'est pour ça que maintenant, je cherche ailleurs..."

Malgré une formation de façadier puis de coffreur, son expérience de la pénibilité et des relations professionnelles ont été rédhibitoires par rapport au bâtiment.

Nestor a objectivement moins d'atouts que Roger pour entrer sur le marché du travail. Son niveau scolaire semble particulièrement faible. Après sa sortie du système scolaire, les intervenants sociaux ont fait corps autour de lui pour l'amener à consentir à une affiliation à la catégorie de travailleur handicapé. Son faible niveau fait aussi barrage par rapport à l'entrée en formation qualifiante.

Cadet d'une fratrie de trois, il vit toujours chez ses parents de milieu social modeste et ne semble pas particulièrement pressé de prendre son indépendance.

Lors de notre dernière rencontre, il vient de terminer une période de dix-huit mois d'activité en peinture dans le parc social, sur un chantier-école. Le retour au chômage lui semble de l'ordre du normal.

Son effort d'intégration sur le chantier-école pendant plus d'une année justifie une certaine bienveillance. Les derniers échanges avec son conseiller ont scellé un accord tacite : Nestor peut maintenant s'accorder légitimement un laps de temps assez long au chômage.

Dans l'immédiat, son attentisme par rapport au marché de l'emploi se confond avec l'idée de retrouver une copine et le rêve de partir en voyage : "(Rire)... Ça va être un peu dur pour trouver du travail et trouver quelqu'un d'autre (une autre fille). Je vais de temps en temps sur le site de Pôle emploi chez moi. Mais je cherche ailleurs (le ton n'est pas du tout convaincant sur son engagement)... Je suis né ici et je n'ai jamais eu l'occasion de voyager, de voir d'autres endroits. Même si je sais que ce ne sera pas forcément mieux ailleurs (Il semble l'avoir entendu d'un intervenant social), j'aimerais voyager sur les îles, par exemple à la Réunion. Les îles, c'est reposant, il n’y aura pas une tour HLM (habitation à loyer modéré) en face de chez moi. Et puis, la mer ça adoucit ..."

La confusion entre la question de l'emploi, le rêve du voyage, l'espoir de trouver une nouvelle copine semble mettre à distance, au moins provisoirement, la question du marché du travail.

Les institutions considèrent l'insertion professionnelle de Nestor comme particulièrement improbable, dans l'immédiat, compte tenu de son niveau proche de l'illettrisme, de son absence de qualification, de ses récits fantaisistes. 
Nestor entretient cette relative mansuétude en contribuant aux bonnes relations avec ses conseillers. Implicitement, les rapports avec ses parents justifient aussi sa faible ambition vers l'autonomie: "Ce n'est pas que mes parents ne veulent pas que je parte, mais mon père préfere mille fois que j'aie un travail sûr pour être certain que je puisse payer le loyer! »

Et comme " le travail sûr " n'est ni pour demain, ni sans doute pour après-demain, Nestor devrait se maintenir " en insertion » encore pendant quelques années...

Les attentistes naviguent entre plusieurs carrières. Ils recherchent du travail dans des rythmes discontinus, se relâchent à certains moments et jouent "à l'élastique » dans leur rapport au marché du travail.

On perçoit, chez Nestor comme chez Roger, combien la confrontation à la pénibilité lors des premières expériences professionnelles a joué un rôle prépondérant dans leur attentisme d'aujourd'hui. Chez l'un comme chez l'autre, le besoin de rêver et la " carrière" (Becker, 1985 ; Mucchielli, 2013) dans les petits trafics contribuent aussi à la mise à distance du marché du travail. Tenir le marché de l'emploi à distance permet aussi de se maintenir dans un rapport au temps au jour le jour, qui facilite une certaine insouciance.

Le scénario le plus probable pour Nestor, c'est de rejoindre ultérieurement " les empêchés ", ceux qui ont intériorisé leur handicap à cause de nombreux désavantages, Roger, quand à lui, semble disposer de plus de ressources pour accéder au monde des "autonomes».

\section{La position des « hyper volontaristes »}

La quatrième position regroupe ceux qui tiennent un discours hyper volontariste et qui sont suspendus de manière ostentatoire à un certain idéal de l'engagement dans la recherche d'emploi. Il s'agit pour eux de mettre en avant, comme essentielles, les attitudes personnelles par rapport au marché du travail.

Quand nous le rencontrons pour la première fois, Sylvain met alors un point d'honneur à montrer son engagement dans son rapport au marché du travail : "Si je ne tombe jamais dans le découragement malgré le chômage, c'est que je me suis toujours obligé à rester dans le circuit de la recherche d'emploi. Régulièrement, je participe à des forums organisés par Pôle emploi ou par la Chambre des Métiers. C'est l'occasion de faire des candidatures spontanées, de voir des patrons et de discuter avec eux..."

Il reprend un certain discours sur la notion d'aires de mobilité et sur les compétences transversales pour s'adapter à l'évolution du marché du travail local et se saisir de toutes les opportunités : "Le directeur de Peugeot PSA a dit l'autre jour qu'il cherche parfois des col- 
laborateurs qualifiés et quill n'en trouve pas, que l'on peut répondre aux attentes des entreprises même si on n'a pas le bon diplôme. On n'a pas le bon diplôme, mais on a des aptitudes et des qualités et on peut s'adapter à l'activité pour répondre aux besoins de l'entreprise."

Sylvain tente d'apposer à son blason les attributs du discours managérial : être proactif, maintenir une activité de veille par rapport au marché de l'emploi et anticiper.

Le discours d'Armand est assez proche : "Il faut savoir se vendre auprès des employeurs. Si vous vous contentez d'envoyer votre $C V$ et votre lettre de motivation, ça n'est pas suffisant, elle risque de finir à la poubelle ou de se perdre dans un dossier. Non, il faut se déplacer et rencontrer l'employeur!... Et là, vous aurez un premier contact et plus de chances parce que vous pouvez avancer vos arguments, il s'agit de mettre tous les atouts de votre côté. Peut-être que l'employeur dira quil est complet pour l'instant, mais quand il aura besoin de vos services, il se rappellera de vous et vous appellera!..."

La recherche d'emploi prend une tournure essentialiste : ce n'est plus seulement sa force de travail, ou ses compétences que l'on doit mettre en avant. La recherche d'emploi équivaut, en quelque sorte, à être le commercial de soi-même.

Ces zélotes se signalent aussi par une certaine familiarisation avec un langage qui montre que l'on est dans le mouvement. Armand : "Je n’hésite pas à prendre des initiatives, récemment, j'ai balancé mon $C V$ au CHU (Centre hospitalier universitaire) et aussi au Futuroscope."

Nous avons rencontré Jean-Jacques pour la première fois sur son lieu de travail. Il est aide-cuisinier en emploi aidé dans un restaurant social. Son discours s'évertuait à nous convertir : "Dans la recherche d'emploi, il faut toujours être sur le qui-vive! Il faut se rendre à Pôle emploi tous les jours pour regarder les offres, c'est important aussi. Il y a beaucoup de gens qui ne le font pas et qui passent à côté d'une offre, à côté de l'emploi... Et donc, ce qui compte d'abord, c'est la motivation... Même si tu n'as pas de qualification, si tu es capable de le faire, il n'y a pas de raison que tu ne trouves pas du travail..."

La motivation et l'implication personnelle seraient susceptibles de résoudre la faiblesse du marché du travail et l'absence de qualification.

L'externalité par rapport à la réalité des contextes des entreprises favorise la reproduction d'un discours confiant, idéal, mais assez déconnecté de l'activité. Ainsi, Armand reprend à son compte, et avec une certaine délectation, le discours de son conseiller : "C'est vrai que l'emploi peut commencer par un remplacement, mais ça peut être évolutif après... Si l'entreprise est satisfaite, si le salarié se dépense et quil fait du bon boulot, sil montre son envie d'avancer, c'est sûr que l'employeur va le récompenser et déclencher ensuite un $C D D$ et par la suite le $C D I$ !... Enfin, moi je me bats aujourd'hui pour ça et j'espère qu'un jour ça va payer !... Parce que, je ne vais quand même pas me battre non plus toute ma vie !..."

Il s'agit de se donner à voir comme un bon demandeur d'emploi et de revendiquer une valeur sociale positive (Goffman, 1974). 
Au début de notre enquête. Sylvain, Jean-Jacques et Armand apparaissent comme les plus clairement dans cette posture.

Leurs discours expriment une idéalisation, par rapport aux normes des dispositifs d'insertion, plus ou moins alimentée par les institutions auprès desquelles ils s'évertuent à faire la preuve de leur loyauté.

Comme l'indique Denis Castra (2003), ces normes sont assez déconnectées des domaines d'activités vers lesquels sont assignées ces populations : la restauration, le bâtiment, les espaces verts, le nettoyage, etc.

Ce sont ceux qui ont eu le plus d'opportunités d'embauche mais qui ont également accumulé le plus d'échecs dans les phases d'intégration en entreprise. Le plus souvent, les tentatives d'intégration en emploi ont échoué dans les semaines ou les mois qui ont suivi l'embauche.

Au-delà de la fréquente mauvaise qualité de l'emploi, la reconstruction de leurs expériences en entreprises témoigne d'une difficulté particulière à appréhender les environnements professionnels, à décoder suffisamment le caractère du jeu relationnel. Sylvain, après son départ d'une enseigne de la restauration rapide, déclare :

"On m'a dit à l'embauche: "Vous aurez à faire face à des périodes de chauffe, mais on forme une équipe et on va construire quelque chose ensemble." En fait, ça n'est pas du tout comme ça. Je me confronte toujours au même ressenti, que ces endroits ne sont pas faits pour moi..."

Il ne s'agit pas simplement d'appréhender l'organisation technique de l'activité. Chaque entreprise correspond aussi à une sorte de matrice relationnelle, un système de communication et d'échanges dans lequel l'individu doit assurer son propre décodage, entre les règles formelles et le fonctionnement implicite, entre la hiérarchie et les formes de leadership, entre le travail prescrit et le travail réel. Cela suppose aussi une construction mentale de la situation en termes de saisie d'opportunités (Sainsaulieu, Francfort, Osty, Uhalde, 1995).

Ces jeunes ne semblent guère en mesure de prendre du recul dans l'interprétation de leurs échecs. Jean-Jacques, après son départ d'une société internationale de restauration rapide :

"Ils mont rappelé pour faire la dernière semaine de la période d'essai, mais je n'y suis pas allé parce que, pour moi, c'était une perte de temps, je savais pertinemment que ça ne marcherait pas! Je ne voulais pas me risquer! Je ne voulais pas rester dans une entreprise où je savais bien que j'irais droit dans le mur!"

Il s'ensuit une tendance marquée à la culpabilité et une perte importante de l'estime de soi. 
Cette idéalisation exacerbe aussi une certaine individualisation de leur rapport à l'emploi qui prend une tonalité exclusive, souvent au détriment des autres dimensions de leur existence.

Cette position sur le marché de l'emploi est généralement soumise à une certaine usure. A la fin de l'enquête, le discours de Jean-Jacques est nettement moins volontariste : "Il n'y a pas de travail et je paie aussi le fait d'avoir quitté mon emploi. Du coup, c'est plus compliqué car sur l'agglomération, les entreprises en restauration se connaissent et mon ancien patron fait barrage... Dans les mois qui viennent, je vais me retrouver à nouveau au RSA (revenu social d'activité) et dans une situation financière difficile. Ça va être compliqué, mais je ne peux rien y faire!..."

Cette idéalisation correspond le plus souvent à une étape de leur " carrière " de chômeur et après quelques années, un certain désenchantement apparaît progressivement. Les expériences du marché du travail et des emplois précaires déconstruisent peu à peu les représentations initiales et obligent à en rabattre.

\section{La position des « empêchés »}

La plupart des jeunes à bas niveau de qualification ont, avec l'expérience, une certaine conscience de leur position défavorable par rapport au marché du travail. A la fin de l'enquête, ils sont encore 18 sur 23 à demeurer hors CDI, sur des positions instables professionnellement et socialement, 7,5 années en moyenne après la sortie du système scolaire.

Mais les empêchés ont particulièrement intériorisé leur handicap à cause de nombreux désavantages : impossibilité d'accéder à la qualification en raison de leur trop faible niveau scolaire, problèmes d'autonomie en matière de déplacements, précarité et problèmes sociaux, faible expérience en entreprise...

Depuis leur sortie du système scolaire, entre chômage et emplois précaires, ils ont l'impression que leur situation s'est progressivement dégradée et se sentent aujourd'hui particulièrement discriminés par rapport aux salariés qui bénéficient encore de relatives protections. Cela se traduit par un sentiment d'impuissance particulièrement marqué.

Le témoignage de Magda sur son parcours professionnel traduit ce processus " d'empêchement ». Voici des séquences de ce qu'elle nous a confié lors d'une même rencontre :

"Après mon apprentissage dans la restauration chez ce traiteur, j'aurais voulu rester, mais il n'y avait pas de boulot. Et puis, comme apprentie, je ne leur coûtais rien, alors ils ne prenaient que des stagiaires ou que des gens en apprentissage..." 
"Eh bien, j'aurais voulu rester dans cette crèche, parce que j'aime beaucoup tout ce qui est contact avec les enfants, mais c'était un Contrat Emploi-Solidarité d'un an, renouvelable un an, et après il n'y a rien à faire pour rester... "

"J'ai fait un stage comme agent de service à la maison de retraite à $M$. Et là aussi, ça mia bien plu. Ils cherchaient à embaucher, mais je n'avais pas de voiture."

"Après, j’ai fait un remplacement à la restauration au CHU, ça a duré deux jours, mais ils ne m’ont pas rappelée après..."

"De toute façon, maintenant c'est dur de trouver un emploi fixe : je sais que les chefs d'entreprise, une fois qu'ils voient une femme avec enfants, ils ne cherchent pas vraiment à comprendre... Ils pensent que les enfants vont être malades, que l'on va s'arrêter. Du coup, ça freine beaucoup pour trouver du travail !..."

Les obstacles apparaissent quasiment insurmontables et Magda se sent glisser, année après année, vers l'assistanat. La crainte de l'étiquetage " de cas social » et ses aspirations de femme qui tient à se montrer dynamique l'obligent à se maintenir comme postulante sur le marché du travail. Elle est assez consciente de l'importance de l'expérience de l'emploi d'un point de vue identitaire : "Il y en a qui aiment bien rester chez eux, mais moi, je n'y arrive pas, je ne suis pas une femme au foyer !... J'ai besoin de bouger, de sortir, de voir du monde, d'être active, de me sentir utile et de ne pas me faire entretenir par un homme. (rire)..."

Conjointement, elle a le sentiment que le marché du travail devient de plus en plus inaccessible, tout en ayant bien du mal à objectiver ce qui fait obstacle à son insertion professionnelle. Comme d'autres, elle est assez convaincue de l'inutilité de creuser cette question douloureuse.

Elle a intériorisé l'expérience du chômage récurrent comme le continuum de son échec scolaire. L'un et l'autre relèvent de la même mise à l'écart et comme d'une fatalité, dont il convient plutôt de prendre acte...

Cette fatalité est aussi alimentée par l'expérience douloureuse d'une certaine indifférence de la part des employeurs. Farah : "Des fois, ils répondent à la demande d'emploi, mais souvent non. C'est vraiment la galère !..."

Dans un certain souci de rationalisation et pour limiter le sentiment d'échec, leurs discours mettent alors en avant certains éléments pour en oublier d'autres, plus difficiles à appréhender.

Magda, comme Farah, fait d'abord allusion à l'absence du permis de conduire. Magda : "Le permis, c'est indispensable, mais ça fait cinq fois que je rate le code... Je ne sais pas ce que j'ai, mais je n'arrive pas à me le mettre dans la tête! ... "

Comme l'indique Olivier Masclet (2002), le document rose représente une institution et les échecs trop fréquents ont souvent laissé des souvenirs cuisants. Si le permis offre, à l'évidence, une certaine autonomie et un atout pour trouver un emploi, il symbolise 
aussi une certaine affirmation de soi, l'accès à une certaine normalité souvent exacerbée par l'expérience de l'échec scolaire et l'expérience institutionnelle.

Le deuxième élément mis en avant est souvent leur propre coût salarial. A l'instar de Magda, il est souvent fait allusion à la concurrence de main-d'œuvre qui ne coûte rien : "Je n'ai pas pu rester au magasin; ils prenaient surtout des stagiaires qui ne coûtaient rien et ils n'embauchaient pas."

En particulier dans les activités de services peu qualifiées, un certain nombre d'employeurs ont recours à un volant de stagiaires qui représente parfois un quart de leur effectif (Glaymann, 2015).

Pour des raisons diverses, un certain nombre de ces jeunes sont trop âgés au moment où ils envisagent de suivre une formation en alternance, par exemple un apprentissage. Ils se trouvent alors dans une position défavorable par rapport à des candidats plus jeunes qui coûtent moins cher.

Comme d'autres dans la même situation, Magda a bien conscience aujourd'hui des représentations négatives par rapport aux jeunes mères isolées et sans qualification.

Finalement, entre l'absence de permis et de qualification professionnelle, soumise à la précarité avec la charge d'enfants en bas âge, c'est l'accumulation de tous ces obstacles qui relègue progressivement Magda vers une certaine forme d'assistanat.

Au-delà, ces jeunes font rarement allusion ${ }^{5}$ à leur absence de qualification comme obstacle premier.

Farah : "Eh bien, il faut s'activer pour trouver du travail, quand on cherche, on trouve! C'est ce qu'on dit! Après, si on n'a vraiment pas de chance, on met plus de temps!"

Il y a, en l'occurrence, une certaine forme de déni dans leurs discours, alimenté par un certain fatalisme : à quoi bon s'attacher, dans l'immédiat, à cette idée de qualification puisque l'on n'a pas les moyens d'y accéder, compte tenu de son faible niveau scolaire et des problèmes matériels que cela poserait?

Sous la pression des institutions et en compensation, il y a par moments une certaine survalorisation de l'importance de la motivation comme la clé pour trouver du travail.

Le déni par rapport à l'impact de la qualification pour sortir du chômage est présent aussi s'agissant du poids de leurs origines :

Farah : "Il y a les gens en haut et nous on est en bas, ça n'est pas grave, ça ne me gêne pas... Et puis, de toute façon, on n'a pas le choix, on doit évoluer avec les moyens du bord. "

5. A contrario, quand ils ont intégré un domaine d'activité, ils évoquent assez souvent l'intérêt de la formation qualifiante pour faire progresser leur carrière. 
Pointer ses origines sociales quand on est issu du bas de l'échelle sociale revient sans doute à pointer sa famille et à contrevenir à une certaine logique de l'honneur.

En revanche, "les empêchés " de notre groupe pointent souvent les étrangers comme responsables de la discrimination qu'ils subiraient. La figure de l'étranger est alors convoquée pour donner corps à un sentiment d'abandon dans l'espace social et mieux comprendre et être mieux compris par un certain discours d'extrême droite.

Magda : "Les Africains, eux, on les aide et nous, on ne nous aide pas, c'est n'importe quoi! L'assistante sociale préfere aider les noirs que nous, c'est l'horreur! Ce n'est pas que je sois raciste, mais ce n'est pas normal cette préférence pour les étrangers. Les noires? Il faut voir comment elles s'habillent, elles ont de la classe, elles sont en Chanel, en Louis Vuitton, elles ont tout le temps de la marque, alors que nous... Et quand on va aux Restos du cour, qui c'est qu'on voit? C'est encore elles... C'est humiliant!"

\section{La position des " autonomes »}

La sixième position correspond aussi à des jeunes qui sont investis dans la recherche d'emploi, mais dont le rapport au marché du travail apparaît plus cohérent et plus lucide en comparaison avec " les hyper volontaristes " et "les attentistes » évoqués précédemment.

Malgré la précarité, ils ne sont pas autant obnubilés par la question du chômage et de l'emploi et s'autorisent des phases de relatifs relâchements entre des périodes de recherche d'emploi.

Le rapport moins exclusif à l'insertion professionnelle favorise un certain pragmatisme dans l'articulation entre l'emploi, qui permet de disposer de quelques ressources pour vivre, et l'expérience du travail pour se réaliser en tant qu'individu.

Antoine : "En fait, pour l'instant, je n'ai pas de projet professionnel précis, il me faut un travail pour manger... Après, quand on aura le choix, je pourrai me permettre de dire ce que j'ai envie de faire !..."

Les enjeux de l'accès à l'emploi n'altèrent pas d'autres investissements, en particulier dans des relations amicales et une vie sociale autour de rencontres informelles. Antoine : "Dans la journée et le soir, j'essaie de bouger, d'aller voir des amis. Pour la plupart, ce sont des copains du lycée et on est resté en contact. Ce sont des amis sur qui je peux compter. Souvent, on prend un verre, on mange quelque chose et on fume un joint. Ça me change les idées et c'est mieux que de rester à réfléchir tout seul..." 
Face à la pression institutionnelle et au vide des périodes de chômage, ces relations leur permettent de se réapproprier un peu de leur existence : "Et puis, c'est vrai que ce sont des amis qui sont dans la même situation que moi. C'est vrai qu'on se comprend quand on en parle, on a vécu un peu les mêmes choses..." (Antoine)

Ces personnes sont alors moins suspendues à la norme du projet des dispositifs d'insertion, moins dépendantes des institutions comme les Missions Locales, les structures d'insertion, etc. Elles conservent une certaine conscience de leur projet de vie. Cette vie sociale, apparemment hors de la sphère du travail, favorise leur capacité à se projeter dans l'avenir et leur fournit une certaine énergie pour leur recherche d'emploi. Il s'agit en quelque sorte d'un effet vertueux.

Au-delà, ces relations permettent aussi de mutualiser les expériences sur le marché du travail, une certaine veille par rapport aux opportunités et une entraide en matière de recherche d'emploi.

Antoine : "Je vais aller voir la semaine prochaine pour une formation de vendeur en boutique. Dans cette société, ils forment des gens pour cette activité et si ça se passe bien, on peut sortir avec un diplôme. J'ai appris cela par une amie qui est également intéressée. On va y aller ensemble et on va voir ce que ça donne..."

Cette sociabilité fait partie intégrante du mode de vie d'Antoine, d'Anaïs et de Vanina. Elle n'est pas accessible à tous : ainsi, depuis son adolescence, Jean-Jacques (groupe des « hyper-volontaristes » évoqué plus haut) a une attitude de défiance marquée et il ne reçoit personne chez lui.

Comme nous l'avons mentionné précédemment, chaque contexte de travail représente une sorte de matrice relationnelle dans laquelle il convient d'être capable d'interagir, non seulement du point de vue des contingences de la production, mais également dans la vie sociale singulière que représente chaque contexte de travail.

Antoine a su se faire coopter par ses collègues de travail de la communauté de communes où il effectue des remplacements à la voirie : "Ça ña pas été facile au début, parce quils sont bien plus âgés que moi. Mais je m'entends vraiment très bien avec tous et il y a une bonne ambiance. Et c'est ça aussi qui me plaît là-bas. Chaque fois qu'il y a besoin de quelqu'un, mes collègues poussent ma candidature et ils disent au chef: "c'est Antoine, le petit jeune qu'il faut prendre". Je compte sur eux pour avoir encore du travail..."

Plus les chômeurs sont orientés vers des contrats aidés dans le secteur non marchand et vers des activités « d'insertion » peu efficientes du point de vue de l'insertion professionnelle, comme le confirme l'INSEE (Aeberhardt, Crusson, Pommier, 2011), plus le mode de recherche d'emploi devient stéréotypé et contribue aussi à l'affaiblissement de cette population.

La standardisation des services pour l'emploi prend souvent implicitement acte de l'absence de qualification de ces populations et de la faiblesse de leurs expériences pro- 
fessionnelles. Elle naturalise, auprès des employeurs eux-mêmes, leur appartenance à la masse des chômeurs sans grande valeur. Le CV devient ainsi une sorte d'objet transitionnel $^{6}$ dont la seule fonction est de rassurer le chômeur lui-même ; la recherche d'emploi prend alors une dimension pathétique (Legrand, Saielli, 2013).

Oscar: "Je suis allé porter mon CV chez un paysagiste dont les bureaux sont à peine à $100 \mathrm{~m}$ de chez moi. Il a lu mon $C V$ et à un moment, il en a eu marre, il en a fait une boule qu'il a envoyée dans sa poubelle devant moi !... Il ne s'est pas gêné et ça ne m’a pas trop plu !.. »

Les autonomes doivent rompre avec une certaine routine en matière de recherche d'emploi, et c'est ce que semble avoir compris, à sa manière, Vanina, 26 ans, qui cherche directement auprès des entreprises dans le nettoyage, la restauration et les services auprès des personnes âgées : "J’ai commencé par m’inscrire à Pôle emploi mais quand il y avait une offre et que je postulais, je n'avais jamais de réponse et je n'arrivais à rien... Ça a duré comme ça assez longtemps, ce qui fait que maintenant je fais du porte-à-porte. Je suis une battante!"

Pour Vanina ou Antoine, il ne s'agit pas de faire fi de l'ensemble des institutions mais plutôt de développer des stratégies alternatives en prenant appui sur leurs expériences, sur leurs relations personnelles et sur leur famille. Ils peuvent alors espérer trouver la bonne distance avec les institutions et articuler, avec une certaine intelligence, ce qui relève de leur vie privée et de l'intervention publique, de leur rapport au marché du travail et de leur projet de vie. La recherche d'emploi semble plus respectueuse des autres sphères de l'existence. Elle gagne aussi en pertinence et en lucidité.

\section{Conclusion}

Les résultats de cette enquête confirment la diversité des modes de vie et des rapports à l'emploi de ces jeunes peu qualifiés. Ils révèlent combien, y compris chez les populations d'origine modeste, il convient de résister à une figure unique du jeune « en insertion " pour revisiter en permanence les catégories dans lesquelles nous serions tentés de les enfermer. La question des rapports à l'emploi alimente facilement des stéréotypes (Borras, 2004).

Nous souhaiterions synthétiser les critères discriminants mis au jour par cette recherche et qui ont motivé la construction de notre typologie.

L'analyse de la position "des déconnectés " pose d'abord la question du repli et de l'engagement par rapport au marché du travail. L'insertion dans le marché du travail est alors renvoyée à plus tard chez " ces déconnectés ", dans une forme d'autant plus idéalisée qu'il n'y a pas de confrontation avec les mondes professionnels. Il semble que, chez ces

6. Au sens de l'objet fétiche qui symbolise et remplace la présence de la mère pour les petits-enfants, en particulier au moment du coucher. 
jeunes, le souvenir de l'échec scolaire alimente assez souvent ce repli, comme si le rapport à l'emploi ne pouvait être que le continuum de cet échec scolaire.

Plus ou moins inconsciemment, ces jeunes redoutent aussi la dureté des activités du marché du travail auxquelles ils sont plus ou moins assignés.

D’une certaine manière, l'engagement exacerbé des « hyper volontaristes " ne semble pas cohérent non plus. Influencés qu'ils sont par un certain discours d'une partie des intermédiaires de l'emploi, pour eux, la motivation et l'implication personnelle pourraient compenser la faiblesse du marché du travail et leur absence de qualification. La recherche d'emploi équivaut à devenir le commercial de soi-même. La loyauté aux institutions dédiées à l'emploi et aux précaires contribue assez souvent à cette débauche d'énergie sur l'objectif de l'emploi. Conjointement, on constate un certain rétrécissement de l'horizon et un trop faible investissement dans les autres dimensions de l'existence.

Ainsi, Jean-Jacques (groupe des " hyper-volontaristes ») n'a aucune relation amicale. Il mène une existence routinière et minimaliste, sans autre loisir que la télévision.

Comme l'indique Sébastien Schehr (1999), ce rétrécissement est d'autant plus dommageable que les contingences de l'accès et de l'intégration en emploi convoquent particulièrement les ressources personnelles, relationnelles de la sphère privée.

La question de la bonne distance avec les intermédiaires pour l'emploi se pose alors, ainsi que celle de la bonne articulation entre le projet professionnel et le projet de vie.

"Les empêchés » ont particulièrement intériorisé leur position défavorable. Objectivement, les jeunes femmes à bas niveau de qualification semblent particulièrement concernées par les difficultés d'accès à l'emploi. A même niveau de qualification, c'est sur ce segment des bas niveaux de qualification que l'écart hommes/femmes est le plus fort en matière de chômage (Guionnet, Neveu, 2009). Ainsi, quand l'insertion s'éternise, c'est sans doute pour elles qu'il y a le plus à redouter.

«Les autonomes » contredisent un des stéréotypes souvent véhiculés chez les intervenants sociaux en charge des chômeurs, des précaires. En effet, le chômage et la précarité ne conduisent pas mécaniquement à l'affaiblissement des liens sociaux et à une perte du sens de l'existence. Les scénarios de vie sont des constructions bien plus complexes. La carrière professionnelle et les rapports au marché de l'emploi sont soumis aux interactions avec les autres espaces sociaux, en particulier avec la sphère familiale et la dynamique relationnelle, qui traversent les rapports au travail et à l'emploi.

Ainsi, prenant appui sur une certaine dynamique relationnelle, les autonomes semblent mieux en mesure de résister aux pressions trop fortes et de trouver la bonne distance avec les institutions. Cette relative distance contribue sans doute aussi à la qualité des interactions et de la collaboration avec les intermédiaires de l'emploi. 
Ces " autonomes " semblent moins suspendus à la norme du projet et plus à même de mutualiser les expériences avec leurs proches. La dynamique relationnelle et certaines formes de débrouillardise leur permettent de se réapproprier leur existence et peuvent constituer des atouts pour s'intégrer dans des activités et en entreprise.

Au-delà, cette dynamique relationnelle contribue à une certaine philosophie de la vie. Après des années dans la précarité, certains se sont particulièrement adaptés à une flexibilité qu'ils ont intégrée dans leur mode de vie : elle semble devenue quasiment une composante de leur identité.

Ainsi, cet article peut inciter les intermédiaires de l'emploi à mieux résister à une certaine standardisation dans les usages du projet d'insertion afin de respecter la singularité de chaque demandeur d'emploi.

La bonne distance avec les demandeurs d'emploi reconnaît alors l'importance de la sphère privée dans l'expérience du chômage, les investissements apparemment hors marché de l'emploi comme des ressources. Il s'agit d'éviter la construction d'un rapport exclusif au marché du travail qui exacerbe souvent des attitudes de repli.

\section{Bibliographie}

Aeberhardt R., Crusson L., Pommier P. (2011), Dossier, Les politiques d'accès à l'emploi en faveur des jeunes : qualifier et accompagner, INSEE.

Becker H.S. (1985), Outsiders. Études de sociologie de la déviance, Métailié, Paris.

Borras I. (2004), "L'orientation des jeunes peu qualifiés, une liberté contrainte ", Formation Emploi, n 88, La Documentation française, pp; 79-95.

Castra D. (2003), L'insertion professionnelle des publics précaires, PUF, Paris.

Glaymann D. (2015), "Quels effets de l'inflation des stages dans l'enseignement supérieur?", Formation Emploi, $\mathrm{n}^{\circ}$ 129, La Documentation française, pp. 5-22.

Goffman E. (1973), La mise en scène de la vie quotidienne. La présentation de soi, Les éditions de Minuit, Paris.

Goffman E. (1974), Les rites d'interaction, Les éditions de minuits, Paris.

Guionnet C., Neveu E. (2009), Féminins/Masculins sociologie du genre, éditions Armand Colin, Paris.

Legrand M.-O, Saielli P. (2013), « La VAE comme espace transitionnel : enjeux et paradoxes ", Formation Emploi, n 122, La Documentation française, pp. 31-50

Masclet O. (2002), " Passer le permis de conduire. La fin de l'adolescence " n 28 Agora. "Débats/Jeunesse». 
Messu M. (1991), Les assistés sociaux. Analyse identitaire d'un groupe social, éditions Privât, Toulouse.

Mucchielli L. et al. (dir.), (2013), Trafics et trafiquants de drogues à Marseille, novembre, Rapport de recherche de l'ORDCS.

Sainsaulieu R., Osty F., Uhalde M. (1995), Les Mondes sociaux de l'entreprise, La Découverte, Paris.

Schehr S. (1999), La vie quotidienne des jeunes chômeurs, PUF, Paris. 